Medicina et Pharmacologia Experimentalis 1967;16:282-288

\title{
Book Reviews - Buchbesprechungen - Livres Nouveaux
}

N. J. Harper und A. B. Simmonds: Advances in Drug Research. Volume I. Academic Press, London 1964. 209 S. 50s.

Die Publikationsreihe «Advances in Drug Research*, deren erster Band vorliegt, wendet sich an Pharmakologen, Chemiker und Biochemiker sowie an Vertreter anderer Fachrichtungen, die an der biologischen und chemischen Wirkungsweise eines Arzneistoffes interessiert sind. Geplant sind umfassende Überblicke nicht nur für gesicherte Tatsachen der Arzneimittelforschung, sondern auch Berichte über die Forschungen auf engen, jüngeren Spezialgebieten. Von den Beiträgen wird erhofft, daß sie Anregung geben und den Weg zu neuen, wertvollen Arbeitshypothesen ebnen.

Der vorliegende Band bringt folgende Beiträge: Doyle und Nayler: Penicilline und verwandte Strukturen; Schauker: Physiologischer Transport von Arzneistoffen; Doyle und Mehta: Antitussiva; Copp: Stoffe mit blockierender Wirkung auf das adrenerge Neuron. — Die Übersichten stammen von inter national anerkannten Autoren, die auf den jeweiligen Gebieten experimentell selbst tätig sind und wertvolle Forschungsergebnisse aufweisen können. Die Darstellungen sind vorzüglich und gewährleisten eine umfassende Information über Fakten, Problematik und völlig offenstehende Fragen der Arzneimittel forschung. Zahlreiche bibliographische Angaben gestatten dem Interessierten ein leichtes Einarbeiten in die einschlägigen Sachgebiete. Die Publikations reihe schafft eine Verbindung zwischen den verschiedenen wissenschaftlichen Disziplinen, die sich mit einem Arzneistoff befassen und kann empfohlen werden. K. Mörsdorf, Bonn. R. H. S. Thompson und E. J. King: Biochemical Disorders in Human Diseases. 2. Auflage. Churchill Ltd., London 1964. 120s.

Die Kenntnis biochemischer Veränderungen, die bestimmten Erkrankungen zugrunde liegen oder eng verbunden mit ihnen auftreten, ist in ständigem Zuwachs begriffen. Die nun vorliegende Neuauflage des bekannten Werkes von Thompson und King kann daher lebhaft begrüßt werden. Von hervorragenden Sachkennern wird in einzelnen Kapiteln unter besonderer Berücksichtigung biochemischer Probleme die Pathophysiologie einzelner Krankheitsbilder abgehandelt; so z. B. Erkrankungen des Intestinaltraktes, des Pankreas, der Leber, des Gallensystems, der Nieren und Nebennieren, der Schilddrüse, des Nervensystems, der Muskeln, des lymphoretikulären Systems, des Urogenitaltraktes sowie die Pathophysiologie der Anaemien, der Blutgerinnung, der Arteriosklerose, der Hypertension, der Bindegewebserkrankungen und der vielseitigen Störungen des intermediären Stoffwechsels. Alle Sachgebiete sind übersichtlich und in klarer Darstellung bearbeitet. Das vorliegende Werk ist für den biochemisch Interessierten von großem Wert; es kann vorzüglich zur Information dienen, nicht zuletzt wegen des gut sortierten Sachverzeichnisses; 
es vermittelt eine Fülle von Einzelbefunden und Anregungen, die dem Verständnis und der Erforschung einzelner Erkrankungen nur dienlich sein

können. $\quad$ K. Mörsdorf, Bonn.

Book Reviews - Buchbesprechungen - Livres Nouveaux 283

N. J. Harper und A. B. Simmonds: Advances in Drug Research, Vol. 2. Academic Press, London 1965. X+205 S. 55s.

Aus der neuen Publikationsreihe «Advances in Drug Research* liegt der zweite Band vor. Das Bestreben dieser Reihe, über Fortschritte der ArzneL· mittelfo $\Gamma$ schung zu informieren, Ivird fortgesetzt. Die BeitГäge des zweiten Ban-des befassen sich mit der W1/8kung von Arzneistoffen auf molekularer Basis. Hierbei wurden in jüngster Zeit bedeutende Ergebnisse vor allem auf dem Gebiet der Catecholamine und damit auf dem adrenergen Sektor, im allge-meinen aber auch im cholinergen Bereich erzielt. Ein Teil der Übersichts-arbeiten basiert auf Vorträgen, die auf dem Chelsea Symposion on the Interaction of Drugs with Receptors 1965 in London gehalten wurden.

Der vorliegende Band bringt folgende Beiträge: Iversen: Hemmung der Noradrenalinaufnahme durch Arzneistoffe; van der Schoot und C reveling: Substrate und Inhibitoren der Dopamin-jffHydroxylase (DBH); Belleau: Be-deutung der Strukturveränderungen von Proteinen für die Regulation von Fermenten und das Verhalten der Rezeptoren; Pratesi und Gra.nct: Struktur und Wirku $\pi \mathrm{g}$ von Catecholaminen und verwandten Verbindungen auf die adrenergen Rezeptoren; Bebbington und Brimblecombe: Muskarinrezeptoren im peripheren und zentralen Nervensystem; Tríggle: 2-Halogenaethylamíne und Rezeptoranalyse. Die genannten Beiträge stehen auf einem hohen Niveau und geben guten Einblick in die Probleme der Molekularpharmakologie. Die begonnene Reihe kann empfohlen werden; sie bietet Anregung und Bereiche-rung für den in der Arzneimittelforschung Tätigen. K. Mörsdorf, Bonn.

Biochemistry of the Retina (1st International Symposium on the Biochemistry of the Retina, London, September 1964) (Biochemie der Netzhaut. 1. Internationales Symposium über die Biochemie der Netzhaut, London, September 1964). Edited by Clive N. Graymore. Supplement to Experimental Eye Research. Academic Press, London and New York 1965. 60 p. 172s. Das vorliegende Buch enthält die Vorträge, angemeldeten und freien Dis-kussionen des 1. Symposiums über die Biochemie der Netzhaut. Der an diesem Gebiet interessierte Leser findet sowohl Arbeiten über experimentelle Untersuchungen als auch Ubersichtsreferate.

Während man sich in früheren Jahren hauptsächlich mit den Problemen der Lichteinwirkung und der damit verbundenen chemischen Prozesse be-schäftigte, tritt jetzt immer mehr die

Erforschung biochemischer Vorgänge der Netzhaut in den Vordergrund. So findet man unter den Vortragsmitteilun-gen Ergebnisse über das Vorkommen von Isozymen, über

Substratabhängigkeit verschiedener Fermente, über topographische Stoffwechselstudien, über die verschiedenen Stoffwechselabbauwege des Kohlenhydratstoffwechsels, über

Untersuchungen der Eiweißsynthese usw. Mehrere Arbeiten beschäftigen sich mit dem

Verhalten des Netzhautstoffwechsels aus entwicklungsgeschichtlicher Sicht.

Eine diesem Symposiumsbericht vergleichbare Zusammenfassung der Ergebnisse biochemischer Untersuchungen der Netzhaut fehlte bis jetzt. Das Erscheinen dieses Buches ist daher sehr zu begrüßen. O. Hockwin, Bonn.

284 Book Reviews - Buchbesprechungen - Livres Nouveaux Vasoaktive Amine - Schockbehandlung/Amines vasoactives - Traite-ment du choc I Vasoactive Amines - Treatment of Shock. 4e Assemblée annuelle de la Société suisse d'Angéiologie en commun avec la Société suisse de Cardiologie, Lausanne, 27 novembre 1965. 
Allocutions présidentielles : J.Cl.Rudler, Geneve, et J.L.Rivier, Lausanne. Rédacteurs I Herausgeber: J. L. Schellíng, Lausanne, et L. K. Widmer, Basel. Bibl. Cardiologica Fasc. 17. S. Karger, Basel/New York 1966. VI + 136 p., 63 fig., 13 tab. Fr./DM 41.—. Im ersten Teil des Bandes wird ein Überblick gegeben über unsere derzeitigen physiologischen und pharmakologischen Kenntnisse kreislaufaktiver Amine. Besondere Berücksichtigung findet dabei der Wirkungsmechanismus der Katecholamine im Bereich des Kreislaufapparates. Der zweite Teil enthält Vorträge, die sich mit der Genese und Behandlung der verschiedenen Schockformen befassen. An zahlreichen klinischen Einzelbeispielen werden Anwendungsmöglichkeiten vasopressorischer Amine demonstriert und das Indikationsgebiet adrenerger Inhibitoren umrissen. Die jeweiligen Hauptreferate sind durch zahlreiche Diskussionsvorträge ergänzt. E. Kreppel, Bonn.

F. Homburger (Ed.): Progress in Experimental Tumor Research, Vol. 8. VI + 297 S., 93 Abb., 36 Tab. Fr./DM 90.- . S. Karger, Basel/New York 1966.

Der neueste Band der Reihe «Progress in Experimental Tumor Research» enthält sieben Artikel, die sich der Zielsetzung der Reihe entsprechend wie-derum mit besonders aktuellen Themen der Tumorforschung beschäftigen: P. M. Gullino (Bethesda) gibt einen Überblick über das interne Milieu von Tumoren, insbesondere über die Beziehungen zwischen Gefäßraum, Intersti-tium und Tumorzelle. - Es folgt ein Artikel von J. M. Vasiliev und V. I. Guel-stein (Moskau) über lokale Wechselbeziehungen zwischen Tumorzellen unter-einander sowie zwischen neoplastischen und normalen Zellen bzw. sonstigen Gewebselementen unter besonderer Berücksichtigung der Verhältnisse in che-misch induzierten Tumoren. - A. Rottino und M. J. Kopac (New York) berichten am Beispiel des spontan auftretenden oder provozierten melanotischen Granuloms von Drosophila melanogaster über Möglichkeiten der Tumor-pathogenese. - - Die beiden folgenden Arbeiten sind der Bedeutung von Viren für die Tumorentstehung gewidmet: J. D. Almeida, A. P. Waterson und E. W. L. Fletcher (London) beschreiben strukturelle Details onkogener Viren; V. De-fendi (Philadelphia) beschäftigt sich mit der durch Viren verursachten Transformation menschlicher oder tierischer Zellen in vitro. - In zwei weiteren Kapiteln werden Probleme der Tumortherapie behandelt: H.Savel (Upton, N.Y.) referiert über die Pharmakologie der die Metaphase arretierenden Pflan-zenalkaloide; A. Engeset (Oslo) bespricht auf Grund eigener experimenteller Untersuchungen den Einfluß von Röntgenstrahlen auf Lymphknoten und Lymphwege. - Den Abschluß bildet ein Gesamtsach- sowie -inhaltsverzeich-nis für alle bisher erschienenen Bände der Serie. An Tumorproblemen inter-essierte klinisch oder experimentelltheoretisch orientierte Mediziner werden in dem Werk wichtige Informationen und Anregungen finden.

K. Karzel, Bonn.

Book Beviews - Buchbesprechungen - Livres Nouveaux 285

The Influence of Industrial and Household Handling on the Composition of Food. Vorträge auf dem 3. Symposium der «Studiengruppe Europäischer Ernährungswissenschaftler» vom 11. bis 13. Mai 1964 in Wageningen. Her-ausgegeben von J. C. Somogyí, Rüschlikon-Zürich. Bibl. Nutritio et Dieta, Fasc. 7. Kargei $\cdot$, Basel/New York 1965. VI + 242 S., 72 Abb., 67 Tab. FiV DM 69.-.

Bei diesem Buche handelt es sich um eine Sammlung von Vorträgen, die auf dem 3. Symposium der «Studiengruppe Europäischer Ernährungswissenschaftler» im Mai 1964 in Wageningen (Holland) gehalten wurden. Im Zeit-alter der weitgehenden industriellen Verarbeitung der Lebensmittel und der Technisierung der Haushalte kommt dem Thema eine besondere 
Bedeutung zu, wie aus den einleitenden Grundsatzartikeln von Somogyi und Cremer, die sich im übrigen mit der Veränderung der Vitamine durch Lagerung, Verarbeitung und Zubereitung befassen, hervorgeht. Mit der Veränderung der Eiweißstoffe und deren Bedeutung beschäftigen sich 3 Artikel, mit der Veränderung von Fetten und Lipiden sogar 7 Arbeiten. 11 bekannte Fachleute der holländischen Studiengruppe nehmen zu aktuellen Problemen der Ernährungswissenschaft mit Vorträgen über Ausbildungs- und statistische Erhebungs-fragen, Nahrungsmittelallergie und -toxikologie, Sortenabhängigkeit des Kar-toffeleiweißgehaltes und gewisse Verdauungsstörungen Stellung.

Bei aller Buntheit der Themen vermitteln die in diesem Band der «Bibliotheca Nutritio et Dieta» gesammelten Vorträge dem Ernährungswissenschaftler und dem in der Praxis Tätigen interessante Ergebnisse der Arbeiten auf die sem wichtigen Gebiete. $\quad 0$. R. Klimmer, Bonn.

Alain L. de Weck/J. R. Frey: Monographs in Allergy, Vol. 1. Immuntolerance to Simple Chemicals - Hypersensitivity to Simple Chemicals as a Model for the Study of Immunological Tolerance. S. Karger, Basel/New York 1966. VI + 142 S., 12 Abb., 8 Tab. Fr./DM 32.- . Eine ebenso eigenartige wie wichtige Erscheinung auf dem Gebiete der Immun-Biologie ist die sogenannte Immuno-Toleranz, d. h. das Fehlen nor-maler Immunreaktionen aufgrund nicht eingetretener Sensibilisierung. Die beiden Verfasser beschäftigen sich in ihrem auf der Auswertung der Literatur und eigener experimenteller Arbeiten basierenden Buch, das als Band 1 der Monographiereihe «Allergie» erschienen ist, mit der Immuno-Toleranz, die gegenüber einfachen chemischen Stoffen auftreten kann. Dies bietet den Vor-teil, daß die Spezifität dieses Vorganges leichter erkennbar und die quantitative Frage überschaubar ist und daß sich das Schicksal des einfachen Antigens verfolgen läßt. Die sofortigen oder verzögerten Überempfindlichkeitsreaktionen gegenüber einfachen chemischen Stoffen mit ihren Sensibilisierungs- und Aus-lösungsphasen werden den Vorgängen der Immuno-Toleranz mit Abschwä-chung oder völligem Fehlen von Reaktionen gegenüber Proteinen, Polysaccha-riden und einfachen chemischen Stoffen bei Mensch und Versuchstier gegen-übergestellt. Die Theorien der Entstehung und die Mechanismen dieses Vorganges werden eingehend geschildert. Das übersichtlich angelegte, sehr gut lesbare und durch Tabellen und Schemata illustrierte Buch erleichtert auch dem auf diesem Spezialgebiet nicht

286 Book Reviews - Buchbesprechungen - Livres Nouveaux bewanderten Leser das Eindringen in die Materie, die fur den Mediziner und Biochemiker gleich wichtig ist, und bietet anhand von mehr als 700 Literaturzitaten Einblick und Zugang zum einschlägigen Schrifttum. Ein interessantes und empfehlenswertes Buch. O. R. Klimmer, Bonn.

Ballistocardiography and Cardiovascular Dynamics. Ed.: Antonius Adńanus Knoop. Amsterdam, April 1965. S. Karger, Basel/New York 1966. VIII + 347 p., 31 tab., 232 fig. Fr./DM 65.—. Auf dem Kongreß 1965, dem schon einige getrennte europäische und ame-rikanische Symposien vorausgegangen waren, standen Themen im Vorder-grund, die auch dem mit dem Verfahren weniger oder überhaupt nicht Ver-trauten Einblick in die diagnostische Valenz der Ballistokardiographie ge-währen können. Diese Referate wurden ergänzt durch eine große Anzahl methodischer und theoretisch-analytischer Arbeiten, die zum Teil unter An-wendung modernster Computertechnik durchgeführt worden waren.

Noch immer ist die Domäne der praktisch-diagnostischen Ballistographie die Früherkennung herzmuskel-ischaemischer Erkrankungen, überhaupt der Bestimmung des «physiologischen Alters» eines Herzens gegenüber dem kalen-darischen Alter seines Trägers. Diese wesentliche 
Kenntnis kann deshalb aus dem Ballistogramm gewonnen werden, weil offenbar nicht nur Informationen über Herzkraft, Schlagvolumen und Auswurfgeschwindigkeit des Blutes aus dem Herzen, sondern auch über mechanische Eigenschaften der blutauf-nehmenden Arterien und anthropologische Relationen, wie Herzgröße und -leistung im Verhältnis zum Körpergewicht, in die Kurve eingehen.

Insoweit stellt die Ballistographie zunächst ein recht globales Diagnosti-kum dar, mit alien theoretischen Bedenklichkeiten, aber auch den praktischen Vorzügen solcher breitstreuender Tests.

Andererseits dienen zahlreiche analytische Untersuchungen, über die berichtet wird, der Aufklärung der Korrelation zwischen Blutauswurf-Modalitäten, peripherem Kreislauf und Ballistokardiogramm. H. Klensch, Bonn. P. Mantegazza and F. Piccinini: Methods in Drug Evaluation. Proceedings of an International Symposion, Milano, Sept. 1965. North Holland, Amsterdam 1966. Guilders 60.- . Infolge der Themenstellung des Symposiums streuen die Inhalte der einzelnen Abhandlungen praktisch über das gesamte Gebiet der pharmakologischen Biologie (z. B. Endokrinologie, cardiovaskuläre Funktionen, autonomes NS, Nar-kotika, Psychopharmaka und viele andere Gebiete therapeutischer Aktualität). Der einheitliche Aspekt ist durch die besondere Betonung methodischer Pro-bleme gegeben.

Natürlich handelt es sich bei dem Buch nicht um eine auch nur einiger-maßen umfassende Aufzählung und Beschreibung pharmakologischer Metho-den, es sei denn, man sieht eine solche Absicht durch entsprechend ausgedehnte Literaturhinweise verwirklicht. Das besondere Anliegen des Buches ist die kritische Bewertung der Anwendbarkeit, Sígnifikanz und Begrenzung der Me-thoden, mit denen die einzelnen Autoren besonders vertraut sind. Diese Sicht

Book Reviews - Buchbesprechügen - Livres Nouveaux 287

wird aber nicht nur im Hinblick auf eine Vervollkommnung des «Screening» abgehandelt, sondern in mehreren Vorträgen steht die Besprechung des Pharma-kons als wissenschaftliches Werkzeug dei- Grundlagenfo $\Gamma$ schung im Vordergrund. Alles in allem vermittelt das Buch einen wertvollen Einblick in die Pro-bleme und Erfolge aktueller Fragestellungen der pharmakologischen For-schung in der besonders anspreche $\pi$ den Art, die Vorträgen meist zu eigen ist.

W.Pritz, Bonn.

R. D. Radeleff: Veterinary Toxicology. Lea \& Febiger, Philadelphia 1964. 314 S., 92 Ill. \$9.00.

Der bekannte a terikanische Veterinärmediziner und Toxikologe Radeleff hat ein handliches Buch über Veterinärtoxikologie geschrieben, an dem ohne Zweifel Bedarf besteht. Kommen doch in den üblichen Toxikologiebüchern die VeГgiftungen bei Haus- und Nutztieren, die in Ländern mit hochentwickelter Tierzucht und technisierter Landwirtschaft eine zunehmende Rolle spielen, meist zu kurz. Schon die Lektüre der ersten drei Kapitel, in denen die Begriffe der allgemeinen und klinischen Toxikologie erklärt und die Präventivmaßnahmen begründet werden, zeigt, daß das Buch sich nicht an einen wissenschaftlich arbeitenden Leserkreis, sondern an Studenten der Tierheilkunde und praktische Tierärzte wendet. In 9 Kapiteln behandelt Verf. Vergiftungen bei Nutz- und Haustie $\Gamma$ en durch die wichtigsten Giftpflanzen Amerikas, die in zahlreichen Photographien dargestellt werden, weiterhin Vergiftungen durch anorganische und organische Giftstoffe, bei denen die Schädlingsbekämpfungs- 
mittel einschließlich der Herbizide naturgemäß im Vordergrund stehen. Dabei werden nach gleichbleibendem Schema Vorkommen der Noxen, klinische Vergiftungssymptome, Sektionsbefunde und therapeutische Maßnahmen behandelt, die Wirkungsmechanismen aber nur soweit besprochen, als dies zum Verständnis der Giftwirkung nötig ist. Autopsiebilder illustrieren das im Text Gesagte. Die letzten drei Kapitel befassen sich mit der chemischen Verunreinigung von Lebensmittein tierischer Herkunft und von Futtermitteln, mit Erkrankungen durch Kampfstoffe und strahlende Materialien. Jedem Kapitel ist ein Verzeichnis der englischsprachigen Literatur angegliedert. Inhalt und Gewicht der einzelnen Kapitel müssen in einem derartigen Buch, das von einem Verf. ge schrieben wurde, unterschiedlich sein. Für den amerikanischen Veterinä $\Gamma$ ist das Buch ohne jeden Zweifel wichtig und wertvoll, es vermittelt aber auch dem Toxikologen und Tierarzt anderer Kontinente interessante und wichtige Erkenntnisse. O. R. Klimmer, Bonn. C. H. Thienes and Th. J. Haley: Clinical Toxicology. Lea \& Febiger, Philadelphia 1964. 661 S., 23 Abb. $\$ 9.50$.

Zur stattlichen Reihe von englischsprachigen Büchern, die sich mit all-gemeiner oder spezieller Toxikologie bzw. mit der Erkennung und Behandlung von Vergiftungen beschäftigen, gehört auch das 1940 erstmalig erschienene und nunmehr in einer vierten, erweiterten und revidierten Auflage vorliegende Buch der beiden amerikanischen «klinischen Pharmakologen und Toxikologen», die ihre große Erfahrung als Hochschullehrer und Berater Ärzten und Studenten der Medizin, Gesundheitsingenieuren und Medizinalbeamten zugänglich machen.

288 Book Reviews - Buchbesprechungen - Livres Nouveaux

Alle praktisch wichtigen Gifte sind nach ihren Hauptangriffspunkten im Orga-nismus in 6 Gruppen geordnet (z. B. Krampfgifte, zentral lähmende Gifte, am peripheren Nervensystem wirksame Gifte, Protoplasmagifte, Blutgifte), da dies nach Meinung der Autoren Diagnose und Behandlung der Vergiftungen erleichtern kann. Viele Giftstoffe mit vielfältigen Angriffspunkten sind in ver-schiedenen Abschnitten aufgeführt. 3 weitere Abschnitte sind den Behandlungsprinzipien und der Diagnose auf Grund klinischer Leitsymptome und chemi-scher Giftnachweise gewidmet, wobei sowohl die Möglichkeiten der Praxis als auch der Klinik und ihrer Laboratorien berücksichtigt wurden. Forensisch-chemische Methoden und biologische Teste dienen der Identifizierung einer sehr großen Reihe wichtiger Gifte. Zu ersteren gehört auch ein Verzeichnis von Spezialreagentien. Dem Leser, der mit Wirkungsmechanismen und Angriffspunkten der Gifte nicht vertraut ist, erleichtert ein alphabetisches Sachregister von 32 Seiten das Auffinden der Gifte im Notfalle. Die Abhandlungen über die einzelnen Gifte sind übersichtlich, leicht faßlich und so knapp abgefaßt, wie dies für das Verständnis des Wirkungsmechanismus, für die Stellung einer Diagnose und die Durchführung einer wirksamen Therapie notwendig ist. Dies und das praktische Taschenformat machen das Buch zu einem ausgezeichneten Lehrmittel für Studierende und zu einem Nothelfer für den auf sich gestellten Arzt. Ich kenne kein gleichwertiges Buch in deutscher Sprache.

0. R. Klimmer, Bonn.

Coming Medical Meetings - Kongreßkalender - Tableau des Congrès

Essen: 25. bis 28. Oktober 1967: 12. Internationaler Phlebologen-Kongreß in Gemeinschaft mit dem Jahreskongreß der Société Française de Phlébologie. Auskunft: Ralf Gerl, Hufelandstraße 55 HI, D-43 Essen (Deutschland). 
Paris : 17, 18 et 19 octobre $1967: 2$ e colloque international sur le mode d'action et le métabolisme des psychotropes phénothiaziniques - 2nd International Symposium on action mechanism and metabolism of psychoactive drugs derived from phenothiazine and structurally related compounds.

Paris : 20 octobre 1967 : Xe journée d'Agressologie.

Renseignements : I. S. Forrest, Veterans Administration Hospital, Palo Alto, Cal. (U.S.A.), ou B.Weber, Hôpital Boucicaut, 78, rue de la Convention, F-75 Paris XVe, France. 